\title{
Estrategias de afrontamiento al estrés en cuidadores de pacientes en hemodiálisis
}

\author{
Lesnay Martínez Rodríguez, Yadira Grau Valdés, Rosamary Rodríguez Umpierre \\ Universidad Central "Marta Abreu" de las Villas. Facultad Ciencias Sociales. Departamento de Psicología. Villa \\ Clara. Cuba
}

\section{Resumen}

La enfermedad renal demanda la asistencia de un cuidador. El cuidador del paciente en hemodiálisis se encuentra expuesto a situaciones de estrés generadas por la actividad de cuidado.

Determinar las estrategias de afrontamiento al estrés en cuidadores de pacientes en hemodiálisis y establecer la correlación entre las estrategias y la variable edad de los cuidadores.

Estudio cuantitativo con alcance descriptivo-correlacional. Se analizaron 48 cuidadores de pacientes hemodializados asistentes al Servicio de hemodiálisis durante el 2016.

Se realizó un análisis descriptivo de las variables: estrategias de afrontamiento, sexo, edad, nivel de escolaridad, años como cuidador y vínculo laboral. Se aplicaron la entrevista estructurada y el Cuestionario de Afrontamiento al Estrés.

Se evidencia un predominio de las mujeres en el desempeño del rol como cuidador para un $81 \%$ de la muestra con una media de 48 años de edad. La focalización en la solución del problema y la reevaluación positiva se reconocen como las estrategias de afrontamiento empleadas con mayor frecuencia por los cuidadores. Se evidencia una correlación débil entre la variable edad y las estrategias de afrontamiento al estrés para un valor $\mathrm{p}<0.05$ y con un nivel de confianza del $95 \%$.

\footnotetext{
Correspondencia:

Lesnay Martínez Rodríguez

Universidad Central "Marta Abreu" de las Villas

Departamento de Psicología

Carretera Camajuaní Km 5 1⁄2 Santa Clara, Villa Clara. Cuba

E-mail: lesnay@uclv.cu
}

Existe un predominio del género femenino en el $81 \%$ de los cuidadores para una media de edad de 48 años y un nivel de escolarización medio superior. Las estrategias de afrontamiento al estrés empleadas se centran en la solución del problema. No existe relación entre las edades de los cuidadores y las estrategias de afrontamiento empleadas.

PALABRAS CLAVE: hemodiálisis; cuidador; edad; estrés; estrategias de afrontamiento.

\section{Stress coping strategies in caregivers of hemodialysis patients}

\begin{abstract}
Kidney disease demand the assistance of a carer. The caregiver of the patient on hemodialysis is exposed to situations of stress generated by the activity of care.

Determine the coping strategies to stress in caregivers of patients in hemodialysis and determine the correlation between the strategies and the variable age of caregivers.
\end{abstract}

Quantitative study with a descriptive- correlational study. We analyzed 48 caregivers of patients attending the hemodialysis service during 2016.

We performed a descriptive analysis of the variables: coping strategies, sex, age, educational level, and years as a caregiver and labor bond. We applied the structured interview and the questionnaire of coping with stress.

There is a predominance of women in the performance of the role as a caregiver for a $81 \%$ of the sample with 
an average of 48 years of age. The focus on the solution of the problem and the positive reevaluation are recognized as the coping strategies most commonly used by caregivers. We found a weak correlation between age and coping strategies to stress to a value of $p<0.05$ and with a confidence level of $95 \%$.

There is a predominance of the female gender in the $81 \%$ of caregivers for an average age of 48 years and a higher average level of schooling.

There is a predominance of the female gender in the $81 \%$ of caregivers for an average age of 48 years and a higher average level of schooling. The stress-coping strategies employed focus on the solution of the problem. There is no relationship between the ages of carers and the coping strategies employed.

KEYWORDS: hemodialysis; caregiver; age; stress; coping strategies.

\section{Introducción}

La Insuficiencia Renal Crónica constituye una importante problemática de salud que afecta severamente a la población mundial. Los pacientes en tratamiento dialítico en América Latina alcanzan una prevalencia de 549 casos por millón de habitantes 1 . En el caso de los pacientes que inician el proceso dialítico como modalidad terapéutica se reconoce una marcada tendencia al incremento expresada en cifras de 190 y 339 casos por millón de habitantes, para América Latina y Estados Unidos respectivamente ${ }^{1}$.

Debido al carácter invalidante del tratamiento, así como las múltiples demandas del cuidado, el paciente en hemodiálisis requiere ser auxiliado por una persona: el cuidador.

Las particularidades del proceder terapéutico demandan al cuidador atenciones especiales que van desde la alimentación hasta el apoyo emocional.

Asumir el rol de cuidador exige modificaciones en el estilo de vida, marcados por cambios a nivel físico, psicológico y social. Garantizar el cuidado del familiar o paciente en hemodiálisis constituye una actividad compleja, exigente y altamente estresante.

El cuidador puede desatender su salud, aislarse, disminuir sus actividades sociales, sufrir cambios en la rela- ción con el paciente y experimentar trastornos sexua$l \mathrm{~s}^{2}$. La pérdida de autoestima, el desgaste emocional severo, las alteraciones del sueño, cefaleas, astenia y cansancio son las principales dificultades que aparecen como consecuencias de la actividad de cuidado ${ }^{3-4}$.

De forma reiterada el cuidador se expone a situaciones de estrés o estresores que han sido denominados como primarios y secundarios ${ }^{5}$. Las acciones que se derivan del cuidado y la atención directa al familiar poseen un carácter primario, lo cual constituye un estresor crónico y altamente significativo para el cuidador $^{5-6}$.

Por su parte los estresores secundarios se asocian a las valoraciones e interpretaciones que realiza el cuidador sobre sus propios recursos y sus relaciones con otros (familiares, amigos, etc.).

En el manejo de las múltiples situaciones de estrés, el cuidador debe desplegar estrategias de afrontamiento para atenuar el impacto emocional que ocasiona la actividad del cuidado. Ello implica una evaluación de sus recursos psicológicos para afrontar los eventos de su vida cotidiana ${ }^{7}$.

El afrontamiento se define como aquellos esfuerzos cognitivos y comportamentales que están en constante proceso de cambio y que se desarrollan para manejar las demandas externas o internas específicas evaluadas como desbordantes para el sujeto ${ }^{7,8,9}$.

El afrontamiento implica un esfuerzo por prevenir, eliminar o debilitar los estresores, se relaciona con la forma en que las personas solucionan o se sobreponen a condiciones altamente estresantes.

Existen a su vez situaciones de estrés imposibles de cambiar, para las cuales el mejor afrontamiento lo constituye aquel que permita minimizar los efectos nocivos de la misma.

El curso crónico de la enfermedad, duración del tratamiento, experiencia en el desempeño del rol, ausencia de apoyo familiar, manifestaciones de sobrecarga y las características de personalidad aparecen como alguno de los factores que influyen en la utilización de una u otra estrategia de afrontamiento ${ }^{10}$.

Por su parte la percepción sobre el cuidado, las capacidades físicas y mentales del cuidador así como las condiciones y recursos para ejecutar la tarea aparecen como factores para disminuir o elevar el estrés. 
El estudio se planteó como objetivos determinar las estrategias de afrontamiento al estrés empleadas en cuidadores de pacientes en hemodiálisis y establecer la correlación entre las estrategias y la variable edad de los cuidadores.

\section{Material y Método}

\section{Diseño}

Se empleó un estudio cuantitativo con alcance descriptivo-correlacional.

\section{Contexto de estudio}

La investigación se desarrolló en la Sala de Hemodiálisis del Hospital Docente "Arnaldo Milián Castro" de la ciudad de Santa Clara durante un periodo de tres meses comprendido desde febrero hasta mayo del 2016. El estudio fue posible mediante un convenio entre la institución hospitalaria y el Departamento de Psicología de la Universidad Central "Marta Abreu" de las Villas.

\section{Muestra}

Se incluyeron 48 cuidadores principales de pacientes en tratamiento renal sustitutivo mediante un muestreo intencional no probabilístico de acuerdo a los criterios de inclusión, exclusión y salida definidos.

- Criterios de inclusión: Cuidadores principales de pacientes en hemodiálisis por más de 6 meses y que deseen participar en la investigación.

- Criterios de exclusión: Cuidadores principales por menos de 6 meses y que no accedan a participar en la investigación

- Criterios de salida: Cuidadores que durante el proceso decidan abandonar la investigación debido al fallecimiento de su familiar o el paciente reciba un trasplante.

\section{Aspectos éticos}

Se estableció el consentimiento informado de los participantes del estudio mediante una entrevista inicial y bajo la firma de un documento elaborado por los investigadores. Se comunicaron los derechos y deberes de los sujetos, horarios de aplicación y pautas de exigencia para los instrumentos a aplicar.

\section{Técnicas}

Se emplearon como instrumentos para la recogida de información:
- Entrevista estructurada: Se empleó como protocolo una entrevista estructurada individual para explorar las variables socio demográficas: sexo, edad, nivel de escolaridad, vínculo laboral y tiempo en el ejercicio del rol de cuidador.

- Cuestionario de Afrontamiento al Estrés (CAE) ${ }^{11}$ : Autoinforme de 42 ítems con una escala de 0-4 diseñado para evaluar siete estrategias básicas de afrontamiento al estrés: Focalizado en la solución del problema (FSP), Autofocalización negativa (AN), Reevaluación positiva (RP), Expresión emocional abierta (EEA), Evitación (E), Búsqueda de apoyo social (BAS) y Religión (R). A cada estrategia de afrontamiento se corresponden de forma aleatoria 6 ítems de la prueba.

\section{Procedimiento estadístico}

Se elaboró una base de datos para recolectar la información y para el procesamiento estadístico de los datos el programa SPSS versión 22. Se realizó un análisis de frecuencia, tomando en consideración la media, desviación estándar de la media y la mediana. A la variable edad y tiempo en el ejercicio del rol se aplicó la prueba de Shapiro-Wilk para evaluar la distribución normal de los datos. Para la correlación entre las variables estrategias de afrontamiento y edad se utilizó un test de Regresión Simple. Como rango de significación estadística se permitió $p<0.05$.

\section{Resultados}

De los 48 cuidadores el $81 \%$ (39) son mujeres y el $19 \%$ (9) hombres, lo que evidencia un elevado predominio del género femenino en el ejercicio del rol de cuidador. La media de edad comprendida para las edades de los sujetos investigados es de 48 años correspondiente con el $31 \%$ de la muestra. (Tabla 1 ).

Tabla 1. Distribución de la muestra por edad.

\begin{tabular}{|lll|}
\hline Rango de edad & Cuidadores & $\%$ \\
\hline $20-30$ años & 6 & $13 \%$ \\
\hline $31-40$ años & 8 & $16 \%$ \\
\hline $41-50$ años & 15 & $31 \%$ \\
\hline $41-50$ años & 11 & $24 \%$ \\
\hline $61-70$ años & 5 & $10 \%$ \\
\hline Más de 70 años & 3 & $6 \%$ \\
\hline
\end{tabular}


El rango de la variable edad oscila entre 24 y 73 años, con una desviación estándar de 13,3862 donde el valor de curtosis estandarizada $(-1,2029)$ se encuentra dentro del rango esperado para datos provenientes de una distribución normal. No se identifican valores atípicos para la muestra.

Se realizó la prueba de Shapiro-Wilk para probar el ajuste a la distribución Normal obteniendo un valor-P de 0,125646 . Por tanto, no se puede rechazar la idea de que los datos de la variable edad provienen de una distribución normal con $95 \%$ de confianza. Los intervalos de confianza del 95,0\% para la media \pm desviación típica: $48,2917+/-3,88695$, de 44,4047 a 52,1786 y para la desviación estándar de 11,1436 a 16,7673. (Gráfico 1).

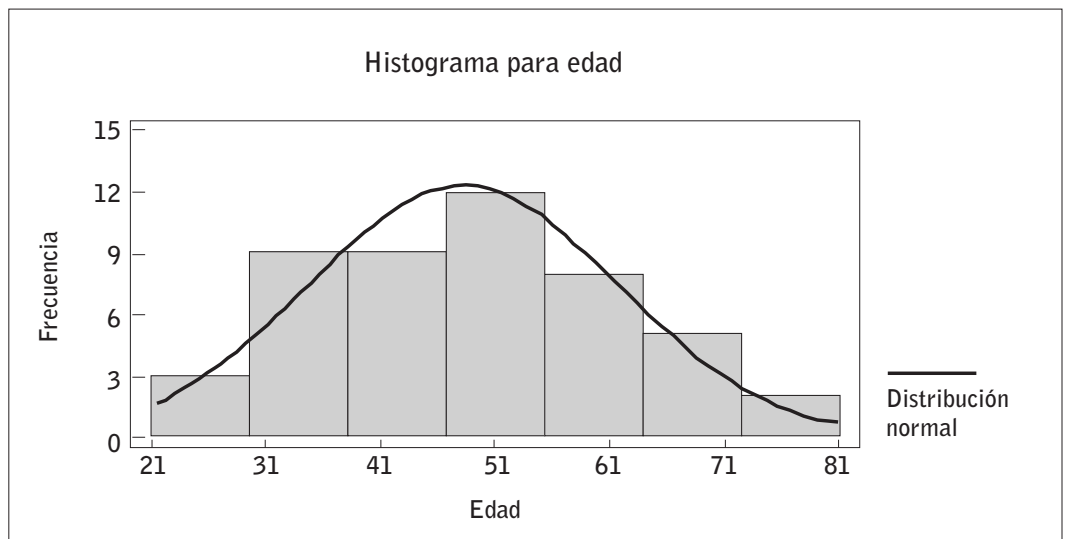

De acuerdo al nivel de escolaridad se evidencia en los cuidadores un predominio del nivel superior medio superior de escolarización. (Gráfico 2).

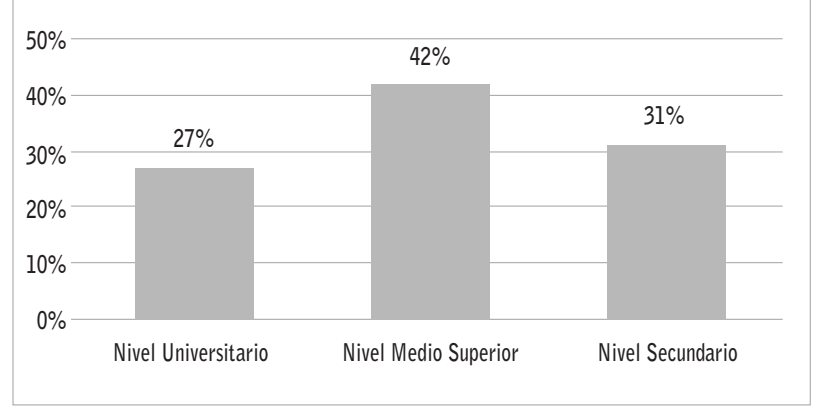

Gráfico 2. Nivel de escolaridad.

En relación con el vínculo laboral se identifica que para un $50 \%$ de la muestra estudiada el desempeño del rol se establece como la actividad prioritaria en los cuidadores, lo cual devino como principal causa para el abandono laboral. (Gráfico 3).

Se empleó el análisis de frecuencia para determinar las estrategias de afrontamiento al estrés empleadas por los cuidadores. (Tabla 3).

Gráfico 1. Histograma para la Distribución normal.

En cuanto al tiempo en el ejercicio del rol como cuidador se identifican diferencias significativas en torno a los años de tratamiento del paciente. Se realizó una prueba de ajuste a la distribución Normal para estos valores y se rechazó la hipótesis con un $95 \%$ de confianza y un valor-P de 7,45299E-7.

Se observó que el $54 \%$ de los cuidadores se han mantenido en el ejercicio del rol por un período de 1 a 10 años, lo cual coincide con el tiempo de diagnóstico y tratamiento de la enfermedad de su familiar. Se destacan además 5 cuidadores que han realizado la actividad de cuidado por más de 20 años. (Tabla 2).

Tabla 2. Distribución de cuidadores de acuerdo a los años en ejercicio del rol.

\begin{tabular}{lll}
\hline Años como cuidador & Cantidad & $\%$ \\
\hline Menos de 1 año & 9 & $19 \%$ \\
\hline De 1 a 10 años & 26 & $54 \%$ \\
\hline De 11 a 20 años & 8 & $17 \%$ \\
\hline Más de 20 años & 5 & $10 \%$ \\
\hline
\end{tabular}

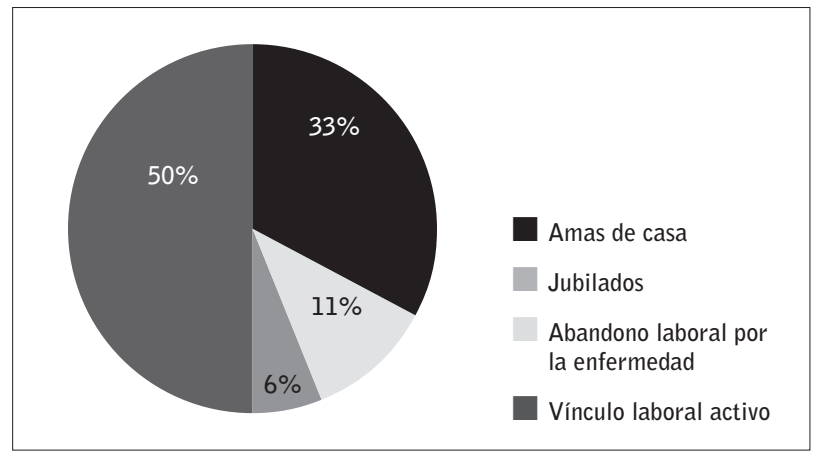

Gráfico 3. Vínculo laboral.

Tabla 3. Distribución de frecuencia de las estrategias de afrontamiento al estrés.

\begin{tabular}{|ll|}
\hline Estrategias de Afrontamiento al estrés & Media \\
\hline Focalizado en la solución del problema & 15,90 \\
\hline Autofocalización negativa & 7,67 \\
\hline Reevaluación positiva & 14,73 \\
\hline Expresión emocional abierta & 5,60 \\
\hline Evitación & 8,71 \\
\hline Búsqueda de apoyo social & 10,69 \\
\hline Religión & 7,67 \\
\hline
\end{tabular}


Para evaluar la correlación entre la variable edad y las estrategias de afrontamiento al estrés se realizó un test de Regresión Simple para el procesamiento de los datos. (Tabla 4).

Tabla 4. Valores en los análisis de correlación.

\begin{tabular}{|lll|}
\hline & $\begin{array}{l}\text { Coeficiente } \\
\text { de correlación }\end{array}$ & $\begin{array}{l}\text { Desviación } \\
\text { estándar }\end{array}$ \\
\hline Autofocalización negativa & 0,191857 & 13,2795 \\
\hline $\begin{array}{l}\text { Búsqueda de apoyo social } \\
\text { Expresión emocional abierta }\end{array}$ & 0,0477406 & 13,5155 \\
\hline Evitación & $-0,0629946$ & 13,504 \\
\hline $\begin{array}{l}\text { Focalizado en la solución } \\
\text { del problema }\end{array}$ & 0,0254342 & 13,529 \\
\hline Reevaluación positiva & $-0,150034$ & 13,3777 \\
\hline Religión & $-0,111202$ & 13,447 \\
\hline
\end{tabular}

De acuerdo con los datos obtenidos podemos afirmar que no existe una relación estadísticamente significativa entre la variable edad y las estrategias de afrontamiento de los cuidadores con un nivel de confianza del $95,0 \%$ o más. Ello indica que para un valor $p<0.05$ existe una relación relativamente débil entre las variables correlacionadas.

\section{Discusión y conclusiones}

Los resultados de nuestra investigación corroboran que el desempeño del rol continúa siendo una actividad predominantemente realizada por las mujeres ${ }^{12,15,16}$ y que se deriva de factores como el género y las expectativas en torno al ejercicio del rol, lo que para el caso de la mujer se asocia directamente como extensión a su rol maternal ${ }^{18}$.

La media de edad para los cuidadores analizados es de 48 años lo cual se corresponde con estudios previos ${ }^{15,16}$ que aseguran la cuarta y sexta década de vida como el período vital en que se concentran las personas que figuran como cuidadores.

En relación con los años en el ejercicio del rol no se identifican diferencias en torno a datos similares para este tipo de muestra ${ }^{12}$. La disponibilidad para realizar el cuidado, los horarios de trabajo flexible o la ausencia de vínculo laboral constituyen condiciones que se ajus$\tan$ a la figura del cuidador ${ }^{18}$. Aquellos que se encuentran vinculados laboralmente reconocen realizar de forma conjunta ambas actividades con altos grados de dificultad debido a la frecuencia y duración del tratamiento, lo que atenta con el cumplimiento de la jornada laboral tradicional.

Se evidencia que la atención total o permanente del cuidador hacia el familiar y las tareas domésticas constituyen las acciones prioritarias para éstos al igual que en otros estudios ${ }^{14,16}$.

El nivel de escolarización de los cuidadores resulta superior al de otras investigaciones encontradas 13,14 donde es válido reconocer para la muestra estudiada mayores niveles de formación e instrucción.

A través del análisis de frecuencia se determinó que la estrategia de afrontamiento, Focalización en la solución del problema y Reevaluación positiva, constituyen las de mayor uso por parte de los cuidadores, resultado que se corresponde con estudios previos que toman como figura de análisis al cuidador ${ }^{17,18}$.

Se evidenció que para la muestra el empleo de esta estrategia se encuentra determinada por la urgencia que requiere el proceso de cuidado. Ello exige que el cuidador afronte la actividad de modo objetivo y práctico, resolviendo los problemas que se le presentan a diario con el fin de proporcionar un mayor bienestar a su familiar. Estos resultados indican que las estrategias se encuentran centradas en la resolución del problema, no así en la emoción.

Es preciso reconocer que no se registran investigaciones antecedentes que ofrezcan resultados en torno a la correlación entre la variable edad y las estrategias de afrontamiento lo cual apreciamos como una limitación, en tanto no podemos establecer comparaciones al respecto.

Se identificó que la relación entre las variables es débil, indicando la ausencia de significación estadística. Ello permite determinar que el empleo de una u otra estrategia de afrontamiento al estrés no se encuentra condicionada por la edad que posean los cuidadores. Estos resultados nos indican que existen otras variables que pueden estar mediando en el empleo de las estrategias de afrontamiento, así como de su eficacia en la solución de situaciones de estrés. 


\section{Agradecimientos}

A los cuidadores del Servicio de Hemodiálisis del Hospital Docente "Arnaldo Milián Castro" de la ciudad de Santa Clara por su participación y disposición en la investigación.

Recibido: 10 febrero 2017

Revisado: 15 marzo 2017

Modificado: 20 abril 2017

Aceptado: 1 mayo 2017

\section{Bibliografía}

1. Anuario Cuba Nefro-Red. 2014. Situación de la Enfermedad Renal Crónica en Cuba. 2014. [acceso 14 octubre 2016]. Disponible en: http://files.sld.cu/ nefrologia/files/2015/09/anuario-nefrologia-2014pagina-web- especialidad.pdf.

2. Arechabala MC, Catoni MI, Palma E, Barrios S. Depresión y autopercepción de la carga del cuidado en pacientes en hemodiálisis y sus cuidadores. Rev Panam Salud Pública. 2011; 30(1):74-9.

3. Astudillo W y Mendinueta C. Necesidades de los cuidadores de pacientes crónicos. Guía de recursos sanitarios y sociales en la fase final de la vida Gipuzkoa $7^{a}$ sección. Madrid. 2008. [acceso 16 octubre 2016]. Disponible en http://www. infogerontologia.com/documents/burnout/pac_ cronico_y_cuidadores.pdf

4. Párraga M. Y ¿Quién cuida a los cuidadores? Enfermería Global. 2005; (6).

5. Saavedra Macías FJ, Bascón MJ, Arias S, García M, Mora D. Cuidadoras de familiares dependientes y salud: influencia de la participación en un taller de control de estrés. Rev. Clínica y Salud. 2013; 24:85-93.

6. Vitaliano PP, Zhang J, Scalan JM. Is caregiving hazardous to one's physical health? A meta-analysis. Psychological Bulletin. 2003; 129: 946-972.

7. Macías MA, Madariaga $C$, Valle $M$, Zambrano J. Estrategias de afrontamiento individual y familiar frente a situaciones de estrés psicológico. Psicología desde el Caribe. 2013; 30 (1): 123-145.

8. Lazarus R, Folkman S. Estrés y procesos cognitivos. Nueva York, Martínez Roca; 1986.

9. Lazarus, R. Estrés y Emoción. Modelo del proceso del estrés, manejo e implicaciones en nuestra salud. ( $1^{\mathrm{a}}$ ed.). Bilbao, Desclée De Brouwer; 2000.

10. Félix A. Significado de cuidar para el cuidador familiar de adultos mayores dependientes en Matamoros, Tamaulipas, México: Una perspectiva de género. [Tesis Doctoral] España: Universidad de Alicante. 2012. [accesol6 octubre 2016]. Disponible en https://rua.ua.es/dspace/ bitstream/10045/27875/1/Tesis_Felix_Aleman.pdf

11. Sandín B, Chorot P. Cuestionario de afrontamiento del estrés (CAE): Desarrollo y validación preliminar. Rev Psicopatología y Psicología Clínica. 2003; 8 (1): 39-54.

12. Achury DM, Castaño HM, Gómez LA, Guevara NM. Calidad de vida de los cuidadores de pacientes con enfermedades crónicas con parcial dependencia. Rev Investigación en Enfermería: Imagen y Desarrollo. 2011; 13 (1): 27-46.

13. Andreu L, Armangué PG, Tamar, L. Aproximación a los cuidados informales de enfermos renales crónicos en el complejo hospitalario de Jaén. Rev Soc Esp Enferm Nefrol. 2007; 10 (2): 127-136.

14. López D, Rodríguez L, Carreño S, Cuenca I, Chaparro L. Cuidadores de pacientes en diálisis peritoneal: experiencia de participar en un programa de habilidad de cuidado. Rev. Enferm Nefrol. 2015; 18 (3): 189-195.

15. Romero E, Maccausland Y, Solórzano L. El cuidador familiar del paciente renal y su calidad de vida, Cartagena (Colombia). Salud Uninorte. 2014; 30 (2): 146-157.

16. Aguilera AI, Castrillo EM, Linares B, Carnero $\mathrm{RM}$, Alonso AC, López P. Análisis del perfil y la sobrecarga del cuidador de pacientes en Diálisis Peritoneal y Hemodiálisis. Rev. Enferm Nefrol. 2016; 19 (4): 359-365. 
17. López J, Rodríguez M. La posibilidad de encontrar sentido en el cuidado de un ser querido con cáncer. Rev. Psicooncología. 2007; 4(1): 111-120.

18. TorrealbaW, Yépez NT. Estrategias de afrontamiento y sobrecarga en cuidadores/as informales de pacientes con insuficiencia renal crónica. [Tesis Pregrado] Colombia: Universidad Centrooccidental Lisandro Alvarado. 2012. [acceso 16 octubre 2016]. Disponible en: http://www.sld.cu/galerias/ pdf/sitios/revsalud/tesis_ana_margarita_espin.pdf.

19. Nasser W, Ibraheem I, Ezzat A, Ebraheim, N. Burden and coping strategies in caregivers of eschizophrenic patients. Journal of American Science. 2011; 7(5): 802-811.

\section{Anexo 1. Consentimiento Informado}

Por medio del presente documento solicitamos su cooperación voluntaria en una investigación que se desarrolla en el hospital universitario "Arnaldo Milián Castro" de Santa Clara, con el objetivo de evaluar si existe relación o no entre las estrategias de afrontamiento y el sentido personal del rol en de cuidadores de pacientes en hemodiálisis.

Usted ha sido seleccionado(a) para conformar nuestra muestra, su participación es voluntaria y de igual forma usted puede tomar la decisión de participar o no. En el caso de no sentirse a gusto con algún procedimiento usted puede negarse 0 abandonar el estudio si así lo desea.

Le garantizamos total anonimato y confidencialidad con la información que pueda ofrecernos, la cual se empleará solo con fines científico-investigativos.

Agradecemos mucho su participación y el valioso aporte que pueda brindarnos, si le surge alguna duda, no vacile en hacer las preguntas que necesite. Si ha comprendido lo anterior y está de acuerdo en participar, le agradecemos no los informa.

Su contribución es muy valiosa para la investigación por tanto agradecemos su colaboración nos encontramos en plena disposición para aclarar dudas o inquietudes que puedan surgir durante la misma.

Declaratoria de voluntariedad

Acepto voluntariamente formar parte de la investigación en calidad de participante. Para ello he sido informado con anterioridad acerca de sus objetivos y conozco los derechos que poseo. 


\section{Anexo 2. Cuestionario de Afrontamiento al estrés (CAE)}

Objetivo: Determinar las estrategias de afrontamiento al estrés.

Instrucciones: En las páginas que siguen se describen formas de pensar y comportarse que la gente suele emplear para afrontar los problemas o situaciones estresantes que ocurren en la vida. Las formas de afrontamiento descritas no son ni buenas ni malas, ni tampoco unas son mejores o peores que otras. Simplemente ciertas personas utilizan unas formas más que otras. Para contestar debe leer con detenimiento cada una de las formas de afrontamiento y recordar en qué medida $\mathrm{Vd}$. la ha utilizado recientemente cuando ha tenido que hacer frente a situaciones de estrés. Rodee con un círculo el número que mejor represente el grado en que empleó cada una de las formas de afrontamiento del estrés que se indican. Aunque este cuestionario a veces hace referencia a una situación 0 problema, tenga en cuenta que esto no quiere decir que $\mathrm{Vd}$. piense en un único acontecimiento, sino más bien en las situaciones o problemas más estresantes vividos recientemente (aproximadamente durante el pasado año).

\begin{tabular}{|c|c|c|c|c|}
\hline 0 & 1 & 2 & 3 & 4 \\
Nunca & Pocas veces & A veces & Frecuentemente & Casi siempre \\
\hline
\end{tabular}

\section{¿Cómo se ha comportado habitualmente ante situaciones de estrés?}

1. Traté de analizar las causas del problema para poder hacerle frente

$$
\begin{array}{lllll}
0 & 1 & 2 & 3 & 4
\end{array}
$$

2. Me convencí de que hiciese lo que hiciese las cosas siempre me saldrían mal

$$
\begin{array}{lllll}
0 & 1 & 2 & 3 & 4
\end{array}
$$

3. Intenté centrarme en los aspectos positivos del problema

$$
\begin{array}{lllll}
0 & 1 & 2 & 3 & 4
\end{array}
$$

4. Descargué mi mal humor con los demás

$$
\begin{array}{lllll}
0 & 1 & 2 & 3 & 4
\end{array}
$$

5. Cuando me venía a la cabeza el problema, trataba de concentrarme en otras cosas

$$
\begin{array}{lllll}
0 & 1 & 2 & 3 & 4
\end{array}
$$

6. Le conté a familiares 0 amigos cómo me sentía

$$
\begin{array}{lllll}
0 & 1 & 2 & 3 & 4
\end{array}
$$

7. Asistí a la Iglesia

$$
\begin{array}{lllll}
0 & 1 & 2 & 3 & 4
\end{array}
$$

8. Traté de solucionar el problema siguiendo unos pasos bien pensados

$$
\begin{array}{lllll}
0 & 1 & 2 & 3 & 4
\end{array}
$$

9. No hice nada concreto puesto que las cosas suelen ser malas

$$
\begin{array}{lllll}
0 & 1 & 2 & 3 & 4
\end{array}
$$

10. Intenté sacar algo positivo del problema

$$
\begin{array}{lllll}
0 & 1 & 2 & 3 & 4
\end{array}
$$

11. Insulté a ciertas personas

$$
\begin{array}{lllll}
0 & 1 & 2 & 3 & 4
\end{array}
$$

12. Me volqué en el trabajo o en otra actividad para olvidarme del problema

$$
\begin{array}{lllll}
0 & 1 & 2 & 3 & 4
\end{array}
$$


13. Pedí consejo a algún pariente 0 amigo para afrontar mejor el problema

$$
\begin{array}{lllll}
0 & 1 & 2 & 3 & 4
\end{array}
$$

14. Pedí ayuda espiritual a algún religioso (sacerdote, etc.)

$$
\begin{array}{lllll}
0 & 1 & 2 & 3 & 4
\end{array}
$$

15. Establecí un plan de actuación y procuré llevarlo a cabo

$$
\begin{array}{lllll}
0 & 1 & 2 & 3 & 4
\end{array}
$$

16. Comprendí que yo fui el principal causante del problema

$$
\begin{array}{lllll}
0 & 1 & 2 & 3 & 4
\end{array}
$$

17. Descubrí que en la vida hay cosas buenas y gente que se preocupa por los demás

$$
\begin{array}{lllll}
0 & 1 & 2 & 3 & 4
\end{array}
$$

18. Me comporté de forma hostil con los demás

$$
\begin{array}{lllll}
0 & 1 & 2 & 3 & 4
\end{array}
$$

19. Salí al cine, a cenar, a «dar una vuelta», etc., para olvidarme del problema

$$
\begin{array}{lllll}
0 & 1 & 2 & 3 & 4
\end{array}
$$

20. Pedí a parientes 0 amigos que me ayudaran a pensar acerca del problema

$$
\begin{array}{lllll}
0 & 1 & 2 & 3 & 4
\end{array}
$$

21. Acudí a la Iglesia para rogar que se solucionase el problema

$$
\begin{array}{lllll}
0 & 1 & 2 & 3 & 4
\end{array}
$$

22. Hablé con las personas implicadas para encontrar una solución al problema

$$
\begin{array}{lllll}
0 & 1 & 2 & 3 & 4
\end{array}
$$

23. Me sentí indefenso/a e incapaz de hacer algo positivo para cambiar la situación

$$
\begin{array}{lllll}
0 & 1 & 2 & 3 & 4
\end{array}
$$

24. Comprendí que otras cosas, diferentes del problema, eran para mí más importantes

$$
\begin{array}{lllll}
0 & 1 & 2 & 3 & 4
\end{array}
$$

25. Agredí a algunas personas

$$
\begin{array}{lllll}
0 & 1 & 2 & 3 & 4
\end{array}
$$

26. Procuré no pensar en el problema

$$
\begin{array}{lllll}
0 & 1 & 2 & 3 & 4
\end{array}
$$

27. Hablé con amigos o familiares para que me tranquilizaran cuando me encontraba mal

$$
\begin{array}{lllll}
0 & 1 & 2 & 3 & 4
\end{array}
$$

28. Tuve fe en que Dios remediaría la situación

$$
\begin{array}{lllll}
0 & 1 & 2 & 3 & 4
\end{array}
$$

29. Hice frente al problema poniendo en marcha varias soluciones concretas

$$
\begin{array}{lllll}
0 & 1 & 2 & 3 & 4
\end{array}
$$

30. Me di cuenta de que por mí mismo no podía hacer nada para resolver el problema

$$
\begin{array}{lllll}
0 & 1 & 2 & 3 & 4
\end{array}
$$

31. Experimenté personalmente eso de que «no hay mal que por bien no venga»

$$
\begin{array}{lllll}
0 & 1 & 2 & 3 & 4
\end{array}
$$


32. Me irrité con alguna gente

$$
\begin{array}{lllll}
0 & 1 & 2 & 3 & 4
\end{array}
$$

33. Practiqué algún deporte para olvidarme del problema

$$
\begin{array}{lllll}
0 & 1 & 2 & 3 & 4
\end{array}
$$

34. Pedí a algún amigo o familiar que me indicara cuál sería el mejor camino a seguir

$$
\begin{array}{lllll}
0 & 1 & 2 & 3 & 4
\end{array}
$$

35. Recé

$$
\begin{array}{lllll}
0 & 1 & 2 & 3 & 4
\end{array}
$$

36. Pensé detenidamente los pasos a seguir para enfrentarme al problema

$$
\begin{array}{lllll}
0 & 1 & 2 & 3 & 4
\end{array}
$$

37. Me resigné a aceptar las cosas como eran

$$
\begin{array}{lllll}
0 & 1 & 2 & 3 & 4
\end{array}
$$

38. Comprobé que, después de todo, las cosas podían haber ocurrido peor

$$
\begin{array}{lllll}
0 & 1 & 2 & 3 & 4
\end{array}
$$

39. Luché y me desahogué expresando mis sentimientos

$$
\begin{array}{lllll}
0 & 1 & 2 & 3 & 4
\end{array}
$$

40. Intenté olvidarme de todo

$$
\begin{array}{lllll}
0 & 1 & 2 & 3 & 4
\end{array}
$$

41. Procuré que algún familiar o amigo me escuchase cuando necesité manifestar mis sentimientos

$$
\begin{array}{lllll}
0 & 1 & 2 & 3 & 4
\end{array}
$$

42. Acudí a la Iglesia para poner velas o rezar

$$
\begin{array}{lllll}
0 & 1 & 2 & 3 & 4
\end{array}
$$

